Louisiana State University

LSU Digital Commons

Faculty Publications

Department of Physics \& Astronomy

$10-7-2007$

\title{
A phenomenological template family for black-hole coalescence waveforms
}

\author{
P. Ajith \\ Gottfried Wilhelm Leibniz Universität Hannover
}

\section{S. Babak}

Max Planck Institute for Gravitational Physics (Albert Einstein Institute)

Y. Chen

Max Planck Institute for Gravitational Physics (Albert Einstein Institute)

M. Hewitson

Gottfried Wilhelm Leibniz Universität Hannover

B. Krishnan

Max Planck Institute for Gravitational Physics (Albert Einstein Institute)

See next page for additional authors

Follow this and additional works at: https://digitalcommons.Isu.edu/physics_astronomy_pubs

\section{Recommended Citation}

Ajith, P., Babak, S., Chen, Y., Hewitson, M., Krishnan, B., Whelan, J., Brügmann, B., Diener, P., Gonzalez, J., Hannam, M., Husa, S., Koppitz, M., Pollney, D., Rezzolla, L., Santamaría, L., Sintes, A., Sperhake, U., \& Thornburg, J. (2007). A phenomenological template family for black-hole coalescence waveforms. Classical and Quantum Gravity, 24 (19) https://doi.org/10.1088/0264-9381/24/19/S31

This Conference Proceeding is brought to you for free and open access by the Department of Physics \& Astronomy at LSU Digital Commons. It has been accepted for inclusion in Faculty Publications by an authorized administrator of LSU Digital Commons. For more information, please contact ir@lsu.edu. 


\section{Authors}

P. Ajith, S. Babak, Y. Chen, M. Hewitson, B. Krishnan, J. T. Whelan, B. Brügmann, P. Diener, J. Gonzalez, M. Hannam, S. Husa, M. Koppitz, D. Pollney, L. Rezzolla, L. Santamaría, A. M. Sintes, U. Sperhake, and J. Thornburg 


\title{
A phenomenological template family for black-hole coalescence waveforms
}

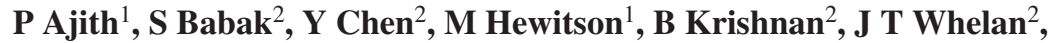 \\ B Brügmann ${ }^{3}$, P Diener ${ }^{4,5}$, J Gonzalez $^{3}$, M Hannam ${ }^{3}$, S Husa $^{3}$, \\ M Koppitz ${ }^{2}$, D Pollney ${ }^{2}$, L Rezzolla ${ }^{2,5}$, L Santamaría ${ }^{3}$, A M Sintes ${ }^{2,6}$, \\ U Sperhake $^{3}$ and J Thornburg ${ }^{2,7}$ \\ ${ }^{1}$ Max-Planck-Institut für Gravitationsphysik (Albert-Einstein-Institut) and Leibniz Universität \\ Hannover, Callinstr. 38, 30167 Hannover, Germany \\ ${ }^{2}$ Max-Planck-Institut für Gravitationsphysik (Albert-Einstein-Institut), Am Mühlenberg 1, \\ 14476 Golm, Germany \\ 3 Theoretisch-Physikalisches Institut, Friedrich Schiller Universität Jena, Max-Wien-Platz 1, \\ 07743 Jena, Germany \\ ${ }^{4}$ Center for Computation and Technology, Louisiana State University, Baton Rouge, LA, USA \\ ${ }^{5}$ Department of Physics and Astronomy, Louisiana State University, Baton Rouge, LA, USA \\ ${ }^{6}$ Departament de Física, Universitat de les Illes Balears, Cra. Valldemossa km 7.5, \\ E-07122 Palma de Mallorca, Spain \\ ${ }^{7}$ School of Mathematics, University of Southampton, Southampton SO17 1BJ, UK
}

Received 30 April 2007, in final form 23 July 2007

Published 19 September 2007

Online at stacks.iop.org/CQG/24/S689

\begin{abstract}
Recent progress in numerical relativity has enabled us to model the nonperturbative merger phase of the binary black-hole coalescence problem. Based on these results, we propose a phenomenological family of waveforms which can model the inspiral, merger and ring-down stages of black-hole coalescence. We also construct a template bank using this family of waveforms and discuss its implementation in the search for signatures of gravitational waves produced by black-hole coalescences in the data of ground-based interferometers. This template bank might enable us to extend the present inspiral searches to highermass binary black-hole systems, i.e., systems with total mass greater than about 80 solar masses, thereby increasing the reach of the current generation of ground-based detectors.
\end{abstract}

PACS number: $04.70 .-\mathrm{s}$

(Some figures in this article are in colour only in the electronic version)

\section{Introduction}

The first generation of ground-based gravitational wave detectors [1-3] are currently operating at unprecedented levels of sensitivity and the LIGO detectors, in particular, have attained their 
design goals over a broad frequency range. The data from these detectors has been used to search for a wide variety of gravitational-wave sources including coalescing binary black-hole systems (see, e.g. [4, 5]). In parallel with these experimental and observational achievements, a series of breakthroughs has occurred in numerical simulations of binary black-hole systems [6-8]. Long-term evolutions of inspiralling black holes that last for several orbits have been obtained with several independent codes [9-16], and accurate gravitational-wave signals have been computed. It is now possible, in principle, to use these numerical-relativity results in astrophysical searches for gravitational waves. However, the high computational cost of these simulations makes it unfeasible to numerically generate all the necessary waveforms to cover the parameter space that needs to be searched. It is therefore necessary, at the present time, to use results from post-Newtonian (PN) theory to extend the waveforms obtained from numerical relativity (NR). The issue of matching NR and PN waveforms for equal-mass binary blackhole systems, and using the numerical-relativity results in gravitational-wave searches, has been considered previously [17-20]. We generalize this to unequal-mass systems and suggest a phenomenological template bank parametrized only by the masses of the two individual black holes. This template bank could be used to search for binary black-hole signals in data from current and future generations of gravitational-wave detectors. Our phenomenological approach is motivated by the work of Buonanno et al [21], but using only physical parameters and generalized to include recent results from numerical relativity.

In this paper, we combine restricted 3.5PN waveforms [22] with results from NR simulations to construct 'hybrid' waveforms for the quasi-circular inspiral of non-spinning binaries with possibly unequal masses. Restricting ourselves to the leading-order quadrupole modes, we find that the hybrid waveforms can be approximated by phenomenological analytical waveforms with fitting factors $\geqslant 0.99$ in the total mass range between 30 and $130 M_{\odot}$ for Initial LIGO [23]. For our analysis, we use numerical waveforms obtained with two independent codes: (i) long waveforms (12 cycles) from equal-mass binaries, provided by the AEI-CCT groups, using their CCATIE code [24] based on the Cactus framework [25] and Carpet mesh-refinement driver [26]; (ii) waveforms from an unequal-mass parameter study presented by the Jena group in [27], which have been obtained with the BAM code [13]. An analysis of these waveforms focusing on ring-down and higher modes has been presented in [28].

The rest of this paper is organized as follows: section 2 summarizes the numerical simulations and how numerical waveforms have been computed. In section 3, hybrid waveforms are produced by matching PN and NR waveforms. In section 4, we propose a family of phenomenological waveforms in the Fourier domain, and study their impact for detection and parameter estimation by computing the fitting factors of the phenomenological waveforms with the hybrid ones. We also parametrize the best-matched phenomenological waveforms in terms of the physical parameters. Section 5 shows the astrophysical range of a search using the full coalescence waveforms and a preliminary comparison with other searches. Finally, section 6 concludes with a summary of our results and plans for future work.

\section{Numerical simulations}

Both the BAM [13] and CCATIE [24] codes are finite-difference mesh-refinement codes solving the Einstein equations within the 'moving puncture' framework [7, 8, 16, 29].

In the wave zone, sufficiently far away from the source, the spacetime metric can be accurately described as a perturbation of a flat background metric; let $h_{a b}$ denote the metric perturbation where $a, b$ denote spacetime indices. Let $t$ be the time coordinate used in the numerical simulation to foliate the spacetime by spatial slices. Working, as usual, in the 
transverse-traceless (TT) gauge, all the information about the radiative degrees of freedom is contained in the spatial part $h_{i j}$ of $h_{a b}$, where $i, j$ denote spatial indices. Let us use a coordinate system $(x, y, z)$ on a spatial slice so that the $z$-axis is along the total angular momentum of the binary system at the starting time. Let $\iota$ be the inclination angle from the $z$-axis, and let $\phi$ be the phase angle and $r$ the radial distance coordinates so that $(r, \iota, \phi)$ are standard spherical coordinates in the wave zone.

Working in the TT gauge, the radiative degrees of freedom in $h_{a b}$ can be written, as usual, in terms of two polarizations $h_{+}$and $h_{\times}$:

$$
h_{i j}=h_{+}\left(\mathbf{e}_{+}\right)_{i j}+h_{\times}\left(\mathbf{e}_{\times}\right)_{i j},
$$

where $\mathbf{e}_{+, \times}$are the usual basis tensors for TT tensors in the wave frame

$$
\left(\mathbf{e}_{+}\right)_{i j}=\hat{\iota}_{i} \hat{\iota}_{j}-\hat{\phi}_{i} \hat{\phi}_{j} \quad \text { and } \quad\left(\mathbf{e}_{\times}\right)_{i j}=\hat{\iota}_{i} \hat{\phi}_{j}+\hat{\iota}_{j} \hat{\phi}_{i} .
$$

Here, $\hat{\imath}$ and $\hat{\phi}$ are the unit vectors in the $\iota$ and $\phi$ directions, respectively. The wave, of course, propagates in the radial direction.

In our numerical simulations, the gravitational waves are extracted by two distinct methods. The first one uses the Newman-Penrose Weyl tensor component $\Psi_{4}$ (see, e.g. [30]) which, in an appropriate gauge, is a measure of the outgoing transverse gravitational radiation in an asymptotically flat spacetime. By measuring that the peeling property (whereby $\Psi_{4}$ falls off as $1 / r$ ) is satisfied we have determined that the gauge we are using does provide a good approximation to within the accuracy required for this study. In the wave zone, it can be written in terms of the complex strain $\mathrm{h}=h_{+}-\mathrm{i} h_{\times}$as [31]

$$
\mathrm{h}=\lim _{r \rightarrow \infty} \int_{0}^{t} \mathrm{~d} t^{\prime} \int_{0}^{t^{\prime}} \mathrm{d} t^{\prime \prime} \Psi_{4}
$$

An alternative method for wave extraction, which has a long history in numerical relativity, determines the waveform via gauge-invariant perturbations of a background Schwarzschild spacetime, via the Zerilli-Moncrief formalism (see [32] for a review). In terms of the even $\left(Q_{\ell m}^{(+)}\right)$and odd $\left(Q_{\ell m}^{(\times)}\right)$parity master functions, the gravitational wave strain amplitude is then given by

$$
\mathrm{h}=\frac{1}{\sqrt{2} r} \sum_{\ell, m}\left(Q_{\ell m}^{+}-\mathrm{i} \int_{-\infty}^{t} Q_{\ell m}^{\times}\left(t^{\prime}\right) \mathrm{d} t^{\prime}\right) Y_{\ell m}^{-2}+\mathcal{O}\left(\frac{1}{r^{2}}\right) .
$$

Results from the BAM code have used the Weyl tensor component $\Psi_{4}$ and equation (3), with the implementation described in [13]. While the CCATIE code computes waveforms adopting both methods, the AEI-CCT waveforms used here were computed using the perturbative extraction and equation (4). Beyond an appropriate extraction radius, the two methods for determining $\mathrm{h}$ are found to agree very well for moving-puncture black-hole evolutions of the type considered here [16].

It is useful to discuss gravitational radiation fields in terms of spin-weighted $s=-2$ spherical harmonics $Y_{\ell m}^{s}$, and in this paper we will only consider the dominant $\ell=2, m= \pm 2$ modes (see [28] for the higher $\ell$ contribution in the unequal-mass case), with basis functions

$$
Y_{2-2}^{-2} \equiv \sqrt{\frac{5}{64 \pi}}(1-\cos \iota)^{2} \mathrm{e}^{-2 \mathrm{i} \phi}, \quad Y_{22}^{-2} \equiv \sqrt{\frac{5}{64 \pi}}(1+\cos \iota)^{2} \mathrm{e}^{2 \mathrm{i} \phi} .
$$

Our 'input' NR waveforms correspond to the projections

$$
\mathrm{h}_{\ell m} \equiv\left\langle Y_{\ell m}^{-2}, \mathrm{~h}\right\rangle=\int_{0}^{2 \pi} \mathrm{d} \phi \int_{0}^{\pi} \mathrm{h} \overline{Y_{\ell m}^{-2}} \sin \iota \mathrm{d} \iota
$$


of the complex strain $\mathrm{h}$, where the bar denotes complex conjugation. In the cases considered here, we have equatorial symmetry so that $\mathrm{h}_{22}=\overline{\mathrm{h}_{2-2}}$, and

$$
\mathrm{h}(t)=\sqrt{\frac{5}{64 \pi}} \mathrm{e}^{2 \mathrm{i} \phi}\left((1+\cos \iota)^{2} \mathrm{~h}_{22}(t)+(1-\cos \iota)^{2} \overline{\mathrm{h}}_{22}(t)\right) .
$$

In practice, we choose $\iota=0$, thus $\mathrm{h}(t)=4 \sqrt{\frac{5}{64 \pi}} \mathrm{h}_{22}(t) \approx 0.631 \mathrm{~h}_{22}(t)$.

\section{Matching post-Newtonian and numerical-relativity waveforms}

Once the PN and NR waveforms are generated, we produce a set of hybrid waveforms by matching them in an overlapping time interval $t_{1} \leqslant t<t_{2}$. The obvious assumption in this procedure is that such an overlapping region exists and that in it both approaches yield the correct waveforms.

Each time-domain waveform $h(t, \boldsymbol{\mu})$ is parametrized by a vector $\boldsymbol{\mu}=\left\{M, \eta, \phi_{0}, t_{0}\right\}$, where $M \equiv m_{1}+m_{2}$ is the total mass of the binary, $\eta \equiv m_{1} m_{2} /\left(m_{1}+m_{2}\right)^{2}$ is the symmetric mass ratio, $\phi_{0}$ is the initial phase and $t_{0}$ is the start time of the waveform. We match the PN waveforms $h_{+, \times}^{\mathrm{PN}}(t, \boldsymbol{\mu})$ and the NR waveforms $h_{+, \times}^{\mathrm{NR}}(t, \nu)^{8}$ by minimizing the squared difference between the respective polarizations, i.e.,

$$
\delta \equiv \min _{\mu, a}\left[\sum_{i=+, \times} \int_{t_{1}}^{t_{2}}\left[h_{i}^{\mathrm{PN}}(t, \boldsymbol{\mu})-a h_{i}^{\mathrm{NR}}(t, \nu)\right]^{2} \mathrm{~d} t\right] .
$$

The minimization is carried over the parameters $\boldsymbol{\mu}$ of the PN waveform and an amplitude scaling factor $a{ }^{9}$ The hybrid waveforms are produced by combining the 'best-matched' PN waveforms and the NR waveforms in the following way:

$$
h_{+, \times}^{\mathrm{hyb}}(t, \nu) \equiv \begin{cases}h_{+, \times}^{\mathrm{PN}}\left(t, \mu_{0}\right) & \text { if } t<t_{1} \\ a_{0} \tau h_{+, \times}^{\mathrm{NR}}(t, \nu)+(1-\tau) h_{+, \times}^{\mathrm{PN}}\left(t, \mu_{0}\right) & \text { if } t_{1} \leqslant t<t_{2} \\ a_{0} h_{+, \times}^{\mathrm{NR}}(t, \nu) & \text { if } \quad t_{2} \leqslant t,\end{cases}
$$

where $\mu_{0}$ and $a_{0}$ denote the values of $\boldsymbol{\mu}$ and $a$ for which $\delta$ is minimum, and $\tau=\left(t-t_{1}\right) /\left(t_{2}-t_{1}\right)$ is a linearly-increasing weighting function, such that $0 \leqslant \tau<1$.

An example set of hybrid waveforms is shown in figure 1. The numerical waveform (black line) from an equal-mass $(\eta=0.25)$ simulation by the AEI-CCT group is matched with a 3.5PN inspiral waveform (red line) over the matching region $100 M \leqslant t<850 M$. The hybrid waveform (dashed line) is constructed by combining the above as per equation (9).

The robustness of the matching procedure can be tested by computing the overlaps between hybrid waveforms constructed with different matching regions. If the overlaps are very high, this can be taken as an indication of the robustness of the matching procedure. A more detailed discussion of this will be presented in [33].

Figure 2 shows the hybrid waveforms of different mass ratios in the Fourier domain. In particular, the panel on the left shows the amplitude of the waveforms in the Fourier domain, while the panel on the right shows the phase. These waveforms are constructed by matching 3.5PN waveforms with the NR waveforms from the unequal-mass $(0.16 \leqslant \eta \leqslant 0.25)$

8 The parameters $\nu$ are taken from the same set as $\boldsymbol{\mu}$, but with different values.

9 The amplitude scaling factor was introduced in order to accommodate possible errors in the amplitude of the NR waveforms due to, for example, the finite radius of the extraction sphere. The best-matched value of $a$, however, was found to be $1 \pm 0.08$. 


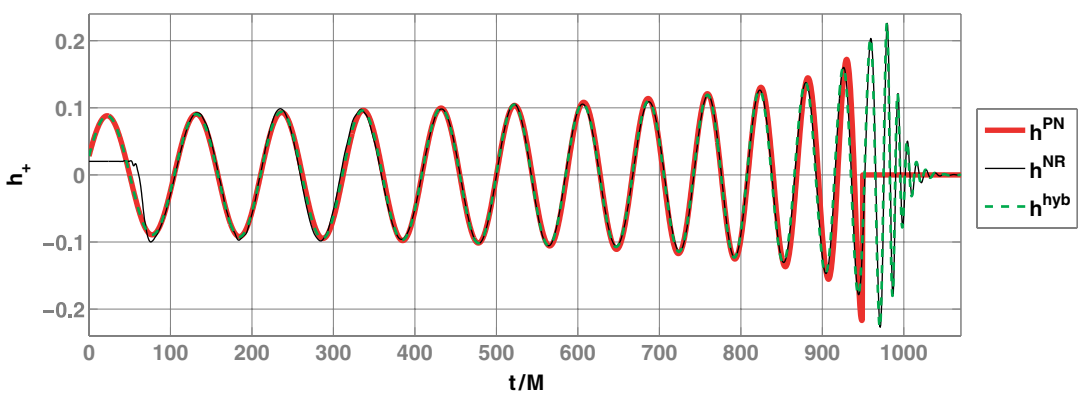

Figure 1. NR waveform (black) from an equal-mass simulation, along with the 'best-matched' 3.5PN waveform (red). The hybrid waveform constructed from the above is also shown (dashed line).
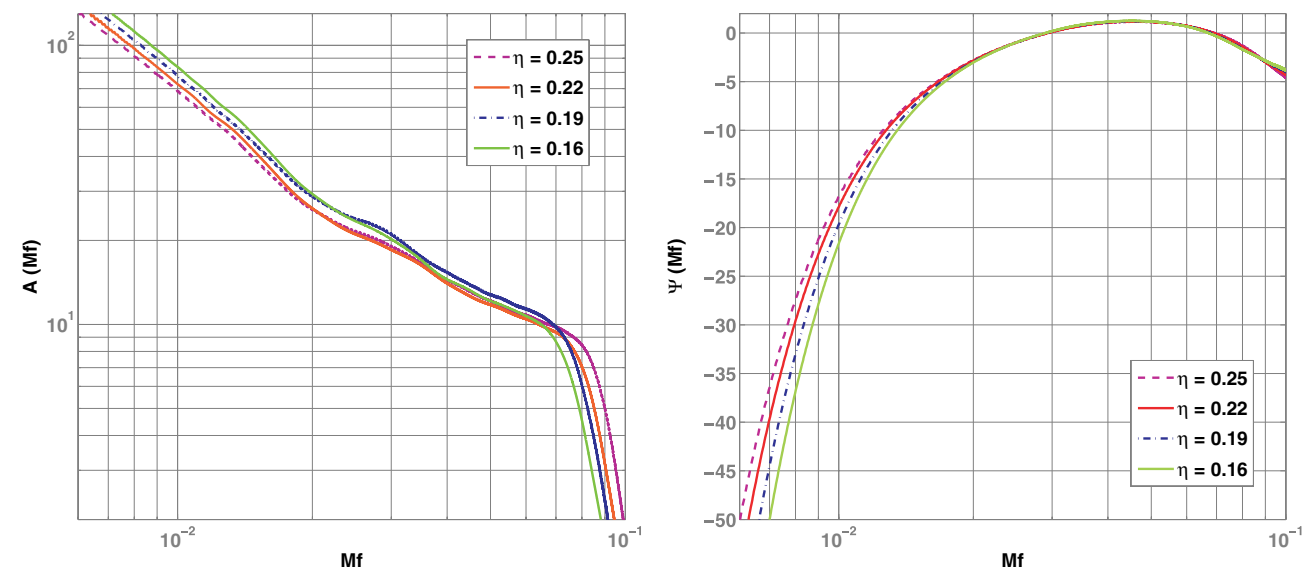

Figure 2. Fourier domain magnitude (left) and phase (right) of the hybrid waveforms. Symmetric mass ratio $\eta$ of each waveform is shown in the legends.

simulations by the Jena group. In the following section, we try to parametrize these Fourier domain waveforms in terms of a set of phenomenological parameters.

\section{The phenomenological template bank}

An obvious issue when using the above-constructed hybrid waveforms directly as detection templates is that it might be computationally very expensive to compute enough NR waveforms to cover the entire parameter space densely enough. In this section, we propose a phenomenological waveform family which has more than $99 \%$ overlaps with the hybrid waveforms in the detection band of the Initial LIGO detectors. We also show how this phenomenological waveform family can be mapped to the physical parameters $(M$ and $\eta)$ so that the template bank, at the end, is two dimensional.

\subsection{Phenomenological waveforms}

We write our phenomenological waveform in the Fourier domain as

$$
u(f) \equiv \mathcal{A}_{\mathrm{eff}}(f) \mathrm{e}^{\mathrm{i} \Psi_{\text {eff }}(f)},
$$




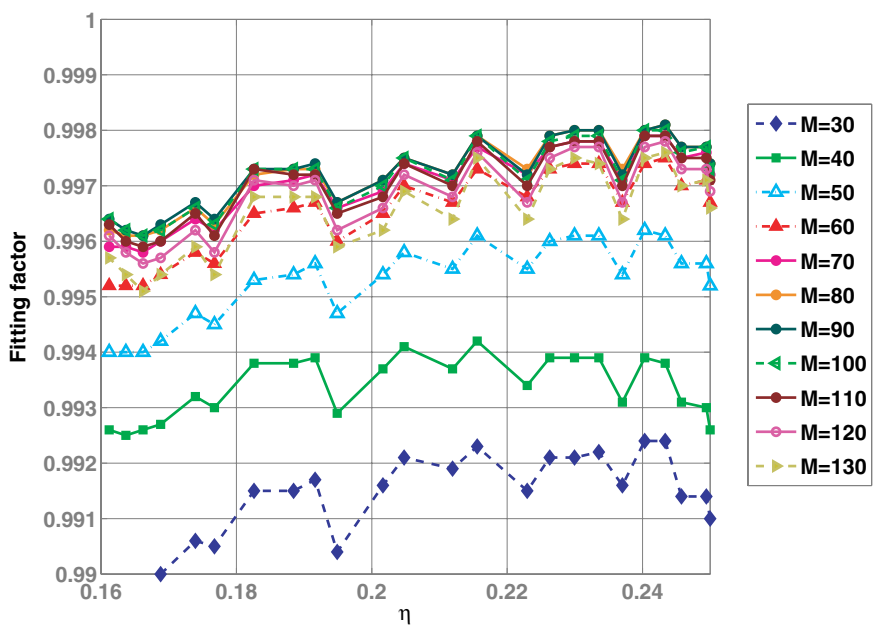

Figure 3. Fitting factors of the hybrid waveforms with the phenomenological waveform family. The horizontal axis shows the symmetric mass ratio of the binary, while different colours/markers correspond to different total masses.

where $\mathcal{A}_{\text {eff }}(f)$ is the amplitude of the waveform in the frequency domain, which we choose to write in terms of a set of 'amplitude parameters' $\alpha=\left\{f_{\text {merg }}, f_{\text {ring }}, f_{\text {cut }}, \sigma\right\}$ as

$$
\mathcal{A}_{\text {eff }}(f) \equiv\left\{\begin{array}{lll}
\left(f / f_{\text {merg }}\right)^{-7 / 6} & \text { if } & f<f_{\text {merg }} \\
\left(f / f_{\text {merg }}\right)^{-2 / 3} & \text { if } & f_{\text {merg }} \leqslant f<f_{\text {ring }} \\
w \mathcal{L}\left(f, f_{\text {ring }}, \sigma\right) & \text { if } & f_{\text {ring }} \leqslant f<f_{\text {cut }} .
\end{array}\right.
$$

In the above expression,

$$
\mathcal{L}\left(f, f_{\text {ring }}, \sigma\right) \equiv\left(\frac{1}{2 \pi}\right) \frac{\sigma}{\left(f-f_{\text {ring }}\right)^{2}+\sigma^{2} / 4}
$$

represents a Lorentzian function of width $\sigma$ centred around $f_{\text {ring }}$. The normalization constant $w$ is chosen in such a way that $\mathcal{A}_{\text {eff }}(f)$ is continuous across the 'transition' frequency $f_{\text {ring }}$, i.e.,

$$
w \equiv \frac{\pi \sigma}{2}\left(\frac{f_{\text {ring }}}{f_{\text {merg }}}\right)^{-2 / 3},
$$

where we use $f_{\text {cut }}$ as the cutoff frequency of the template and $f_{\text {merg }}$ as the frequency at which the power law changes from $f^{-7 / 6}$ to $f^{-2 / 3}$ (as noted previously in [17] for the equal-mass case).

Taking our motivation from the stationary-phase expansion of the gravitational-wave phase, we write the effective phase $\Psi_{\text {eff }}(f)$ as an expansion in powers of $f$.

$$
\Psi_{\text {eff }}(f)=2 \pi f t_{0}+\phi_{0}+\psi_{0} f^{-5 / 3}+\psi_{2} f^{-1}+\psi_{3} f^{-2 / 3}+\psi_{4} f^{-1 / 3}+\psi_{6} f^{1 / 3},
$$

where $t_{0}$ is the time of arrival, $\phi_{0}$ is the frequency-domain phase offset and $\boldsymbol{\beta}=\left\{\psi_{0}, \psi_{2}\right.$, $\left.\psi_{3}, \psi_{4}, \psi_{6}\right\}$ are the 'phase parameters', that is the set of phenomenological parameters describing the phase of the waveform.

The fitting factors [34] of the hybrid waveforms with the family of phenomenological waveforms are shown in figure 3 over the parameter range of $30 \leqslant M / M_{\odot} \leqslant 130$ and $0.16 \leqslant \eta \leqslant 0.25$, using the Initial LIGO noise spectrum. It is quite apparent that the fitting factors are almost always greater than 0.99 , thus underlining the effectiveness of the 


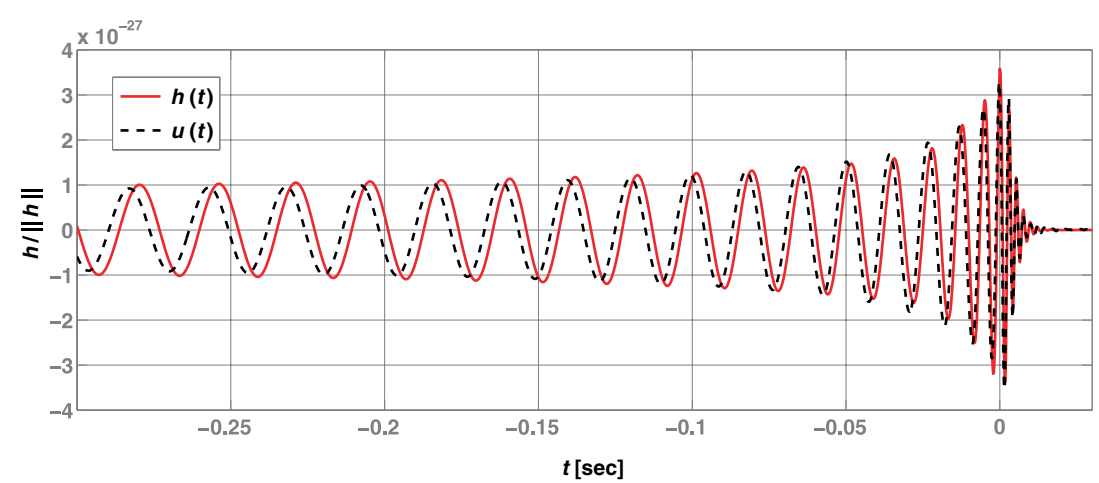

Figure 4. Hybrid waveform $h(t)$ and the best-matched phenomenological waveform $u(t)$ in the time domain for a $M=40 M_{\odot}, \eta=0.25$ binary system. $u(t)$ is computed by taking the inverse Fourier transform of the phenomenological waveform $u(f)$. Both waveforms are normalized with respect to the Initial LIGO noise spectrum.
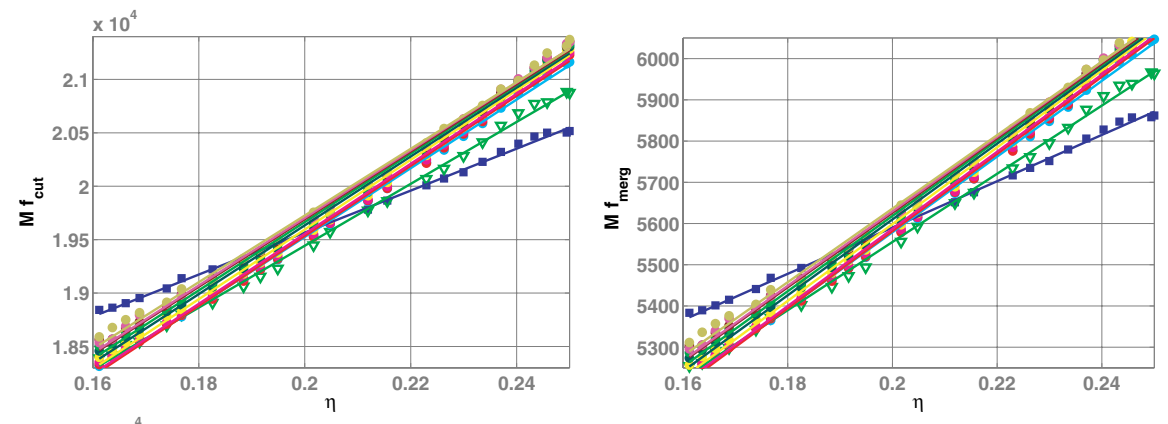

- $M=30$

$\nabla M=40$

- $M=50$

- $M=60$

- $M=70$

- $M=80$
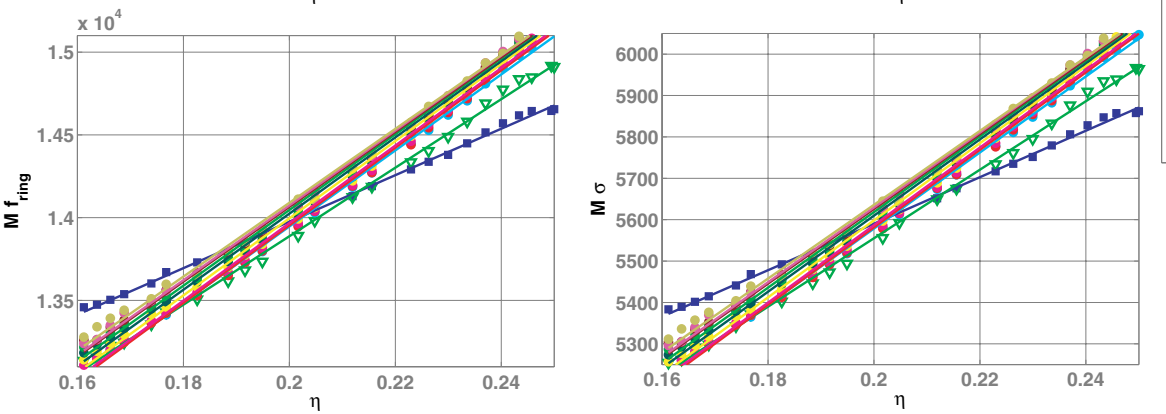

- $M=100$

- $M=100$

- $M=120$

- $M=130$

Figure 5. Best-matched amplitude parameters $\alpha_{\max }$ in terms of the physical parameters of the binary. The horizontal axis shows the symmetric mass ratio of the binary and different colors/markers correspond to different total masses. Linear polynomial fits to the data points are also shown.

phenomenological waveforms in reproducing the hybrid ones. As an example, in figure 4, we plot the hybrid waveform and the best-matched phenomenological waveform from the $M=40 M_{\odot}, \eta=0.25$ binary.

\subsection{From phenomenological to physical parameters}

It is possible to reparametrize the best-matched phenomenological waveforms in terms of the physical parameters of the hybrid waveforms. In figure 5, we plot the amplitude parameters 

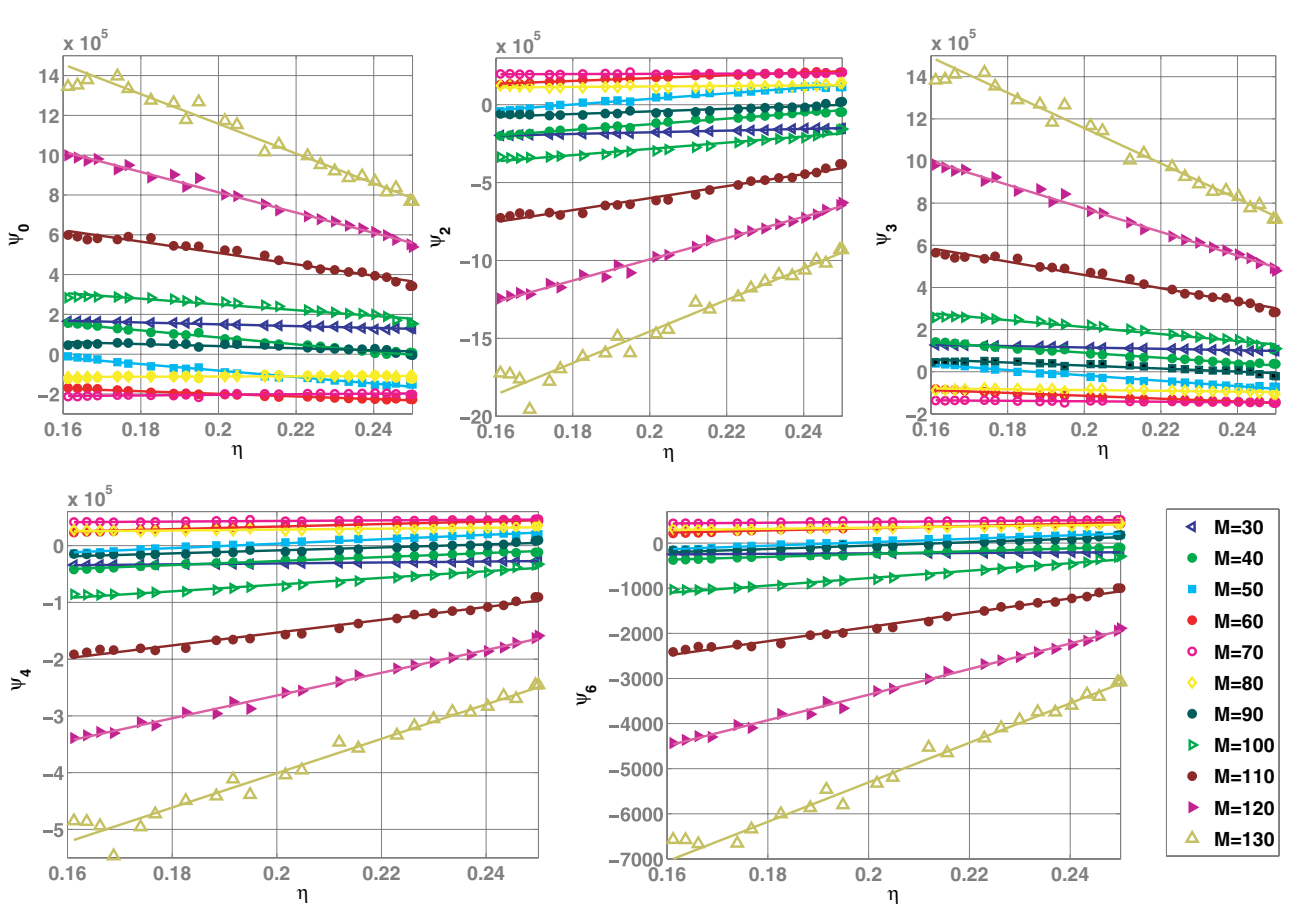

Figure 6. The same as in figure 5 but for the phase parameters $\boldsymbol{\beta}_{\max }$.

$\boldsymbol{\alpha}_{\max }$ of the best-matched phenomenological waveforms against the physical parameters of the binary. Similarly, the phase parameters $\boldsymbol{\beta}_{\max }$ of the best-matched phenomenological waveforms are plotted against the physical parameters of the binary in figure 6 . The linear polynomial fits to the data points serve as a numerical 'look-up table' to go from the physical parameters $\{M, \eta\}$ to the best-matched phenomenological parameters $\left\{\boldsymbol{\alpha}_{\max }, \boldsymbol{\beta}_{\max }\right\}$. Thus, the template bank lives on a two-dimensional manifold (parametrized by $M$ and $\eta$ ) embedded in a higher-dimensional space.

It might be worth stressing that the search will be carried out over $M$ and $\eta$, and not over the phenomenological parameters. The phenomenological parameters $\boldsymbol{\alpha}$ and $\boldsymbol{\beta}$ are constrained by the numerical look-up tables (see figures 5 and 6), and only serve as an intermediate step in generating the template waveforms.

\section{The astrophysical range and comparison with other searches}

The template family proposed in this paper can be used for coherently searching for all the three stages (inspiral, merger and ring-down) of the binary coalescence, thus making this potentially more sensitive than searches which look at the three stages separately. Figure 7 compares the sensitivity of the searches using different template families. What is plotted here is the distance at which an optimally-oriented, equal-mass binary would produce an optimal signal-to-noise ratio (SNR) of 8 at the Initial LIGO noise spectrum. The dotted line corresponds to a search using PN templates truncated at the Schwarzschild innermost stable circular orbit (ISCO), the dashed line corresponds to a search [35] using ring-down templates and the solid line corresponds to a search using all the three stages of the binary coalescence using the template bank proposed here. The horizontal axis reports the total mass of the binary, 


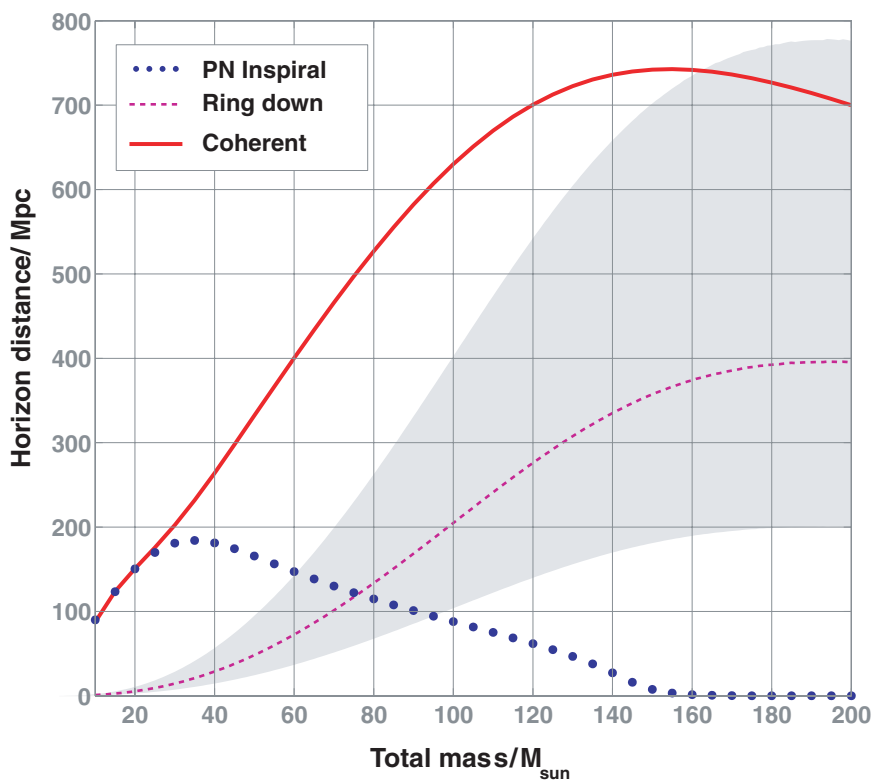

Figure 7. A preliminary assessment of the phenomenological template bank. This figure plots, as a function of the total mass, the distance to an optimally-oriented, equal-mass binary which can produce an optimal SNR of 8 at the Initial LIGO noise spectrum. The dotted line corresponds to a search using PN templates truncated at ISCO, the dashed line corresponds to a search using ring-down templates and the solid line corresponds to a search using the template family proposed in this paper. The ring-down horizon distance is computed assuming that $\epsilon=0.7 \%$ of the black-hole mass is radiated in the ring-down stage. Since the value of this parameter has some amount of uncertainty in it, we have also included the shaded region in the plot corresponding to $0.18 \% \leqslant \epsilon \leqslant 2.7 \%$.

while the vertical axis reports the distance in Mpc. It is quite evident that, for binaries with $50 \leqslant M / M_{\odot} \leqslant 140$, the 'coherent search' using the new template family is considerably more sensitive than any other search considered here. In a forthcoming paper [33], we will quantify and extend this comparison to include other approaches which model all the three stages of black-hole coalescence such as, for example the effective one-body approach [36], or alternatively, combining the results from separate inspiral and ring-down phases using a coincidence analysis (see, e.g. [37]). It is worth pointing out that regardless of the sensitivity, a coherent template bank, such as the one proposed here, is likely to be technically easier to implement than the inspiral-ring-down coincidence analysis. While the method proposed here is just another template bank search, the inspiral-ring-down coincidence would require tuning the individual inspiral and ring-down searches, as well as finding the appropriate coincidence windows and other pipeline parameters.

However, while this looks promising, we emphasize that it is important to treat figure 7 as only a preliminary assessment; fitting factors are not the only consideration for a practical search strategy. It is also very important to consider issues which arise when dealing with real data. For example, false alarms produced by noise artifacts might well determine the true sensitivity of the search, and these artifacts will inevitably be present in real data. This is however beyond the scope of the present work, and further investigation is required before we can properly assess the efficacy of our phenomenological template bank in real-life searches. 


\section{Summary and outlook}

Making use of the recent results from numerical relativity, we have proposed a phenomenological waveform family which can model the inspiral, merger and ring-down stages of binary black-hole coalescence. We first constructed a set of hybrid waveforms by matching the NR waveforms with the analytical PN waveforms. Then, we analytically constructed phenomenological waveforms which approximated the hybrid waveforms. The family of phenomenological waveforms that we propose was found to have fitting factors larger than 0.99 with the hybrid waveforms in the detection band of Initial LIGO. We have also shown how this phenomenological waveform family can be parametrized in terms of the physical parameters ( $M$ and $\eta$ ) of the binary, so that the template bank, at the end, is two dimensional. This phenomenological waveform family can be used to densely cover the parameter space, avoiding the computational cost of generating numerical waveforms at every grid point in the parameter space. We have also compared the sensitivity of a search using this template family with other searches. This search might enable us to extend the mass range of the present inspiral searches to higher mass $\left(>80 M_{\odot}\right)$ systems. In the mass range $50 M_{\odot}$ to $140 M_{\odot}$, this search could be significantly more sensitive than the search using the standard PN inspiral templates and quasi-normal mode ring-down templates.

The numerical 'look-up tables' to go from the physical parameters to the phenomenological parameters (see figures 5 and 6) can be replaced by analytical functions of $M$ and $\eta$. This makes it easier to compute the parameter space metric used for template placement [38] and will be studied in future work. Our plans for future work also include the study of the robustness of the matching procedure used to construct the hybrid waveforms by considering different matching regions and PN waveforms of different order, and of course, to eventually construct a realistic search pipeline which incorporates numerical-relativity waveforms in gravitational wave searches.

\section{Acknowledgments}

The authors thank Lisa Goggin and Steve Fairhurst for help in computing the ring-down horizon distance. NR computations were performed with the Belladonna and Peyote clusters of the Albert Einstein Institute, the Doppler and Kepler clusters of the Jena group and at LRZ Munich and HLRS, Stuttgart. This work was supported in part by DFG grant SFB/Transregio 7 'Gravitational Wave Astronomy'. The Jena group thanks the DEISA Consortium (co-funded by the EU, FP6 project 508830) for support within the DEISA Extreme Computing Initiative (www.deisa.org). AMS gratefully acknowledges the support of the Spanish Ministerio de Educación y Ciencia research project FPA-2004-03666 and the Albert Einstein Institute and the University of Jena for hospitality. PD thanks the Albert Einstein Institute for hospitality. The PN waveforms were generated using the LSC Algorithms Library (LAL), and numerical data-analysis calculations were performed with the aid of the Merlin, Morgane and Zeus clusters of the Albert Einstein Institute.

\section{References}

[1] Waldman S J (for the LIGO Scientific Collaboration) 2006 Class. Quantum Grav. 23 S653-60

[2] Hild S (for the LIGO Scientific Collaboration) 2006 Class. Quantum Grav. 23 S643-51

[3] Acernese F et al 2006 Class. Quantum Grav. 23 S635-42

[4] Abbott B et al (for the LIGO Scientific Collaboration) 2006 Phys. Rev. D 73062001

[5] Abbott B et al (for the LIGO Scientific Collaboration) 2007 Preprint arXiv:0704.3368v2 [gr-qc] 
[6] Pretorius F 2005 Phys. Rev. Lett. 95121101

[7] Campanelli M, Lousto C O, Marronetti P and Zlochower Y 2006 Phys. Rev. Lett. 96111101

[8] Baker J G et al 2006 Phys. Rev. Lett. 96111102

[9] Pretorius F 2006 Class. Quantum Grav. 23 S529-52

[10] Campanelli M, Lousto C O and Zlochower Y 2006 Phys. Rev. D 73061501

[11] Baker J G et al 2006 Phys. Rev. D 73104002

[12] Sperhake U 2006 Preprint gr-qc/0606079

[13] Brügmann B et al 2006 Preprint gr-qc/0610128

[14] Scheel M A et al 2006 Phys. Rev. D 74104006

[15] Herrmann F, Hinder I, Shoemaker D, Laguna P and Matzner R A 2007 Preprint gr-qc/0701143

[16] Koppitz M et al 2007 Preprint gr-qc/0701163

[17] Buonanno A, Cook G B and Pretorius F 2006 Preprint gr-qc/0610122

[18] Baumgarte T et al 2006 Preprint gr-qc/0612100

[19] Baker J G et al 2006 Preprint gr-qc/0612024

[20] Pan Y et al 2007 Preprint gr-qc/0704.1964

[21] Buonanno A, Chen Y and Vallisneri M 2003 Phys. Rev. D 67024016

[22] Blanchet L, Damour T, Esposito-Farese G and Iyer B R 2004 Phys. Rev. Lett. 93091101

[23] Abramovici A A et al 1992 Science 256 325-33

[24] Alcubierre M et al 2003 Phys. Rev. D 67084023

[25] http://www.cactuscode.org

[26] Schnetter E, Hawley S H and Hawke I 2004 Class. Quantum Grav. 211465

[27] González J A et al 2007 Phys. Rev. Lett. 98091101

[28] Berti E et al 2007 Preprint gr-qc/0703053

[29] Hannam M, Husa S, Pollney D, Brügmann B and Murchadha N Ó 2006 Preprint gr-qc/0606099

[30] Stewart J Advanced General Relativity (Cambridge Monographs on Mathematical Physics) (Cambridge: Cambridge University Press)

[31] Teukolsky S A 1973 Astrophys. J. 185635

[32] Nagar A and Rezzolla L 2005 Class. Quantum Grav. 22 R167

Nagar A and Rezzolla L 2006 Class. Quantum Grav. 234297 (erratum)

[33] Ajith P et al 2007 in preparation

[34] Apostolatos T A 1995 Phys. Rev. D 52 605-20

[35] Goggin L M (for the LIGO Scientific Collaboration) 2006 Class. Quantum Grav. 23 S709-13

[36] Buonanno A and Damour T 1999 Phys. Rev. D 59084006

[37] Luna M and Sintes A M 2006 Class. Quantum Grav. 23 3763-82

[38] Owen B J 1996 Phys. Rev. D 53 6749-61 